\title{
Discussion on Informatization Construction of Health Management in Community Health Service
}

\author{
Baiming Jin ${ }^{1, a^{*}}$, Hong Chao ${ }^{1, b}$ and Siyuan Wan ${ }^{1, c}$ \\ ${ }^{1}$ School of Public Health, Qiqihar Medical University, Qiqihar 161006, China \\ ajinbm1520@126.com, b7046376@qq.com, 'wansiyuan0424@126.com \\ * the corresponding author
}

\begin{abstract}
Keyword: Community; Health service; Health management; Informatization.
\end{abstract}
\begin{abstract}
The health management of community health service is a complete process of monitoring and analyzing, evaluating, predicting, preventing, maintaining and developing the health risk factors of the healthy population and the unhealthy population in the community. The implementation of community health management is main pathway to treat disease passively for proactive health management. And health management will be the base rooted in the community, and which aims to improve social fairness, promote community initiative, and solve livelihood problems with full advantage of maximum efforts. In the regional health information platform, through the establishment of health records, regular health examination for the residents of the focus groups, regularly carry out health assessment system, assessment, health intervention can be implemented in the whole process of health management on residents through sharing the regional health information, control the disease occurrence and development, and gradually improve the overall health level the whole crowd.
\end{abstract}

\section{Introduction}

Today, the importance and significance of the development of community health service has no need to repeat, but the direction of development of community health service and strategy, especially the increase in the national health awareness, in the new medical reform has just issued, all focus on something in the background. How to give full play to the role of community health management in community health service in health management and how to develop strategic issues also need to do a clear exposition.

\section{Background of Community Health Service and Health Management}

With the improvement of the living standard of urban residents in China, people's health consciousness is also increasing, and people are no longer satisfied with the traditional medical service mode of "seeking medical treatment before suffering from illness". At the same time, with the rapid development of social economy and the accelerated process of urban population aging, the prevalence of various diseases and elderly diseases has risen, so the demand for health care and improvement has been increasing. The city community health resources allocation structure of our country is not reasonable, and the community health services of medical services of which provided is far lower than the needs of community residents, finally, the city residents have to face the "difficult and expensive" problems. Therefore, the urban community health service in our country must reform the operation mechanism of community medical service, change the traditional medical service model, and establish the community medical service system centered on health management. Health management refers to the overall management process of monitoring, analyzing, evaluating and predicting and preventing the health risk factors of an individual or a population.

The occurrence and development of diseases is undergoing lifestyle long-term accumulation process, the basic mode of health management is through the induction, analysis and control of risk factors causing disease, in order to prevent or control the disease development. 


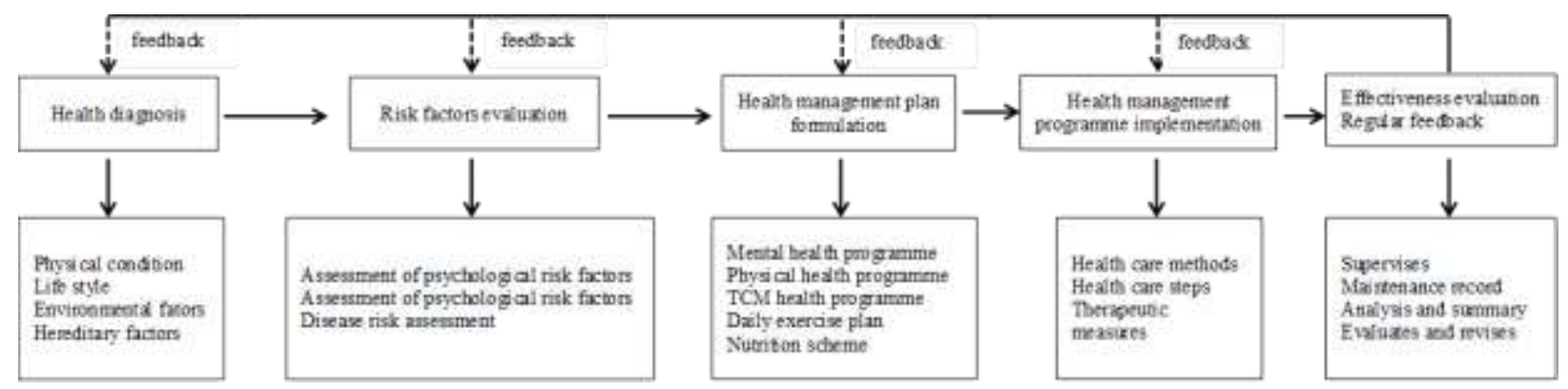

Figure 1. Flow chart of health management for community health service

\section{The Necessity of Information Construction for Health Management of Community Health Service}

Community health service center, as an important service institution of community service, shoulders the important task of meeting the basic medical care and health care needs of community residents. At the same time, as the "nerve terminal" of the medical system throughout the country, it also undertakes the tasks of disease prevention and control and epidemic reporting. Health management from the aspects of heredity, diet, living habits, living environment, occupation behavior, tracking and forecasting, early warning of diseases and various factors affecting the health of the forward-looking concept in all aspects of health interventions, combined with advanced medical care services and information technology to all levels of medical institutions as the basis, to provide scientific and humane system and a full range of health services for the residents, in order to mobilize the enthusiasm of individual and family health, full and effective use of limited medical resources to achieve maximum health improvement, the occurrence and development of disease prevention and control of disease, reduce medical costs, improve the quality of life objective. But at present most of the community health service and health management in health monitoring and management mode have some defects, many community health service management, monitoring and management in the content in the health examination, the lack of health education for pregnant women; in the form of traditional manual management, lack of utilization of community medical information system; treatment based on the static data processing, lack of health monitoring for the whole process.

However, to realize the two-way referral service mode, must be based on the information and data exchange of the community health service center and comprehensive hospital based community health service center must have the modern network technology, communication technology, control technology. This shows that the traditional management model has been unable to meet the growing needs of pregnant women, the use of advanced information systems to achieve imminent community health service health management.

\section{Information Construction of Health Management in Community Health Service}

Raise Awareness and Fully Understand the Deep Meaning of Information Technology. In the construction of community health service informatization, we should consider the function reorganization of community health service center. Information technology cannot be simply understood as the existing electronic business, from "manual" to "computer" service. The meaning of informatization is to make full use of modern information technology and make effective use of information resources. At present, most information systems of community health service center is still in the financial management information system as the focus, which is not completely turned to the patient as the center. With the construction of hospital information system and clinical management, and residents in the field of prevention and health care, medical services and health management information independently, repeated construction and sharing cannot be achieved. Therefore, the health administrative departments should actively coordinate the planning departments, financial departments, scientific research departments and technical suppliers, and jointly develop practical information construction strategy planning and project planning, led by the government. All these involve the reorganization of the government's service functions and cross sectoral cooperation, combining the related business with the public demand. 
Setting Up the Information Platform of Community Health Service to Realize the Refinement of Health Management. On the basis of the region's population, housing and chronic disease census work, establish the health status of the population database, and combined with the urban geographic information system, the establishment of community health service information platform, the development of large screen dispatching system to realize quantitative and qualitative and positioning of health management object. And by the general practitioner team responsible for the management of residents health follow-up, the health situation at any time into the community health service information system, ensure the responsibility of the residents of the region related risk factors of chronic disease, the overall prevalence of information, information and individual residents get the position of timely and accurate update.

At present, in accordance with the grid management mode, using geographic encoding technology can be found in the region, each street, each community, each meter grid in five kinds of chronic disease prevalence and every inhabitant of specific health conditions, to achieve the precise management of chronic disease patients and high risk population.

The Construction of Community Health Service Informatization is Based on the Principle of Human Orientation. In addition to taking into account the needs of community residents for information technology, people who are not familiar with information technology should also be considered. Information technology can be used in a variety of ways, in the community health service center, you can use computers, the Internet, mobile phones, touch-screen, electronic display and other means to achieve information technology services. But for diverse social groups, the provision of information technology services must ensure that everyone is able to use and easy to use. Therefore, in the implementation of the construction of information technology, we should take into account the people who are familiar with and know the information technology, and also take into consideration the people who are not familiar with it and those who do not know it, including the elderly and the disabled. We should fully understand the situation of the people in the service community, such as life, work, social background, community culture, etc.. There are different health needs in different communities. For example, some communities have a higher proportion of elderly people, some communities have some chronic diseases higher than other communities, some communities have fewer local residents, more floating population, and lower cultural quality of the population.

According to the characteristics of different communities, we should adopt the service mode that is suitable for the community residents to grasp the information technology capabilities, and use the platform of community health education to popularize the knowledge of information technology services. Not only to publicize how to use the new technology, but also for the public feedback on new technologies, feedback, publicity, so that information services can quickly get support from residents of the community, and accelerate the process of information. The object of the community health service is the staff of the community, including health, high-risk population and population, the goal is to promote and maintain community health, work in preventive services and medical services are required to pay attention to. Therefore, community health services to community residents as the center for the prevention, community health services, medical care, rehabilitation, health education and family planning guidance demand, completely change the hospital as the center of the original service process.

The embedded information technology in community health service, in addition to replace the manual operation to automation, improve the quality of community health service, the efficiency, the first principle is to health service needs of community residents as the guidance, technical support of community health service process optimization, change "patient" to the community direct delivery health service

Develop Community Health Service Software, Process Reengineering, and Promote Service Optimization. To ensure the health management system and continuous development in the community health service system as one of the basic medical and health management, health education and family planning guidance, rehabilitation of the disabled, the health guidance, community clinics and rehabilitation services and the whole process of community health station of drugs, equipment, equipment, financial and human resources management in the whole process. For each GPS are equipped with "general notebook computer", the establishment of health records, personal life records and electronic medical records for the residents of chronic disease, and chronic disease patients and high-risk population of free Dongcheng District 
residents health card, realize data acquisition, slow disease health information service flow of information to ensure the system, health management and continuity.

The hospital is a knowledge intensive industry, and its business processes are much more complicated than other services. In the past, the hospital management process in China has mainly focused on internal management and cost saving. It is not too convenient to consider the convenience of patient visits. When the former district hospital was transformed into a community health service center, the functions of the existing district hospitals, besides the medical care and treatment of the community residents, also increased the tasks of disease prevention and control and epidemic reporting. The number of personnel can not reach rapid expansion, and to improve service efficiency, it is necessary to integrate resources and process reengineering. For the hospital, it can learn from the ideas and methods of business process reengineering of enterprises, analysis of community health service center of the business process, and re design process by means of modern technology, to improve the service efficiency of the basis of the original resources. In the process of reengineering, the support of information technology will break the traditional management rules and create new ways of work.

Based on the needs of residents' community health service, the results of combing and optimizing business processes are embedded into the information system to realize the standardization and innovation of the service process. The treatment process reengineering and process optimization projects include: Reengineering and optimization of outpatient service process, hospitalization, outpatient services such as logistics, which process is the key to the entire medical process optimization, directly reflects the community health service center service level. The optimized service process, embedded into the specific content of services, to achieve the whole process of the use of network information technology support, ensure all residents are able to obtain convenient, cheap, quick and thoughtful, safe and reliable so as to improve the community health services, community health Level of service.

\section{Summary}

In short, the informatization construction of city community health service center is not only to assist the community health service institutions simply use information technology to daily work, it is a concrete manifestation of the ability of the government in community management and service. The informatization work of community health service center, is to use a variety of information technologies and means, government, community health services and community residents to establish communication channels for service in community, improve the government's social management ability, make the community better service, community residents satisfaction, so as to achieve the continuous improvement of the health level of residents. To strengthen the informatization construction of community health services, the establishment of disease information network system, high speed and high efficiency of the system for disease prevention and control, maternal and child health care system and medical treatment system to improve the ability of the community health service level and respond to public health emergencies, has very important significance.

\section{References}

[1] De L S, Chan T. The development of primary care information technology in the United kingdom [J]. Journal of Ambulatory Care Management, 2008, 31(3):201.

[2] Campbell S M, Scott A, Parker R M, et al. Implementing pay-for-performance in Australian primary care: lessons from the United Kingdom and the United States [J]. Medical Journal of Australia, 2011, 194(4):213-4.

[3] De L S, Tomson C, Harris K, et al. Creatinine fluctuation has a greater effect than the formula to estimate glomerular filtration rate on the prevalence of chronic kidney disease [J]. Nephron Clinical Practice, 2011, 117(3):c213-c224.

[4] Korhonen P E, Kautiainen H, Järvenpää S, et al. Time to change the glomerular filtration rate estimating formula in primary care [J]. European Journal of Internal Medicine, 2012, 23(4):355-357. 